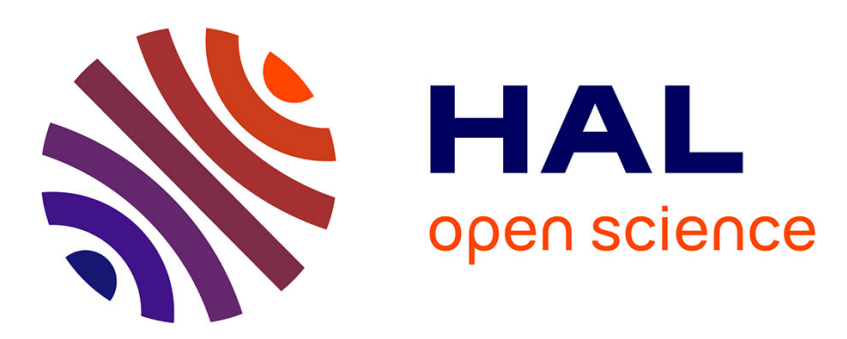

\title{
ATOMIC STRUCTURE OF GRAIN BOUNDARIES AND INTERGRANULAR SEGREGATION
}

\author{
V. Vitek, Gui Wang
}

\section{To cite this version:}

V. Vitek, Gui Wang. ATOMIC STRUCTURE OF GRAIN BOUNDARIES AND INTERGRANULAR SEGREGATION. Journal de Physique Colloques, 1982, 43 (C6), pp.C6-147-C6-161. 10.1051/jphyscol:1982615 . jpa-00222295

\section{HAL Id: jpa-00222295 https://hal.science/jpa-00222295}

Submitted on 1 Jan 1982

HAL is a multi-disciplinary open access archive for the deposit and dissemination of scientific research documents, whether they are published or not. The documents may come from teaching and research institutions in France or abroad, or from public or private research centers.
L'archive ouverte pluridisciplinaire HAL, est destinée au dépôt et à la diffusion de documents scientifiques de niveau recherche, publiés ou non, émanant des établissements d'enseignement et de recherche français ou étrangers, des laboratoires publics ou privés. 
JOURNAL DE PHYSIQUE

Colzoque C6, supplément au n० 12, Tome 43, décembre 1982

page $\mathrm{C} 6-147$

\section{ATOMIC STRUCTURE OF GRAIN BOUNDARIES AND INTERGRANULAR SEGREGATION}

V. Vitek and Gui Jin Wang

Department of Materials Science and Engineering, University of Pennsylvania, PhitadeIphia, PA. 19104, U.S.A.

Résumé.- Nous abordons quelques aspects importants de la structure atomique des joints de grains dans un métal pur et plus particulièrement le modèle de structure des joints quelconques et la multiplicité des structures. On prêsente un schéma empirique de la description des interactions atomiques dans les alliages. Dans le cadre de ce schéma on examine les caractéristiques générales, indépendantes de la structure, de la ségrêgation. Les résultats des calculs atomistiques pour les joints de grains présentant soit un faible taux d'impuretés, soit un taux croissant d'impuretês, sont prêsentés. Les relations entre la structure des joints et la tendance à la sêgrêgation, ainsi que les changements structuraux provoqués par la ségrégation sont discutées à la lumière de ces calculs.

\footnotetext{
Abstract.- Some important aspects of the atomic structure of grain boundaries in pure metals, in particular the structural model of general grain boundaries and the multiplicity of structures, are discussed first. An empirical scheme for the desciption of atomic interactions in alloys is then outlined. Genera1, structure independent, properties of segregation are examined in the framework of this scheme. Results of the atomistic calculations of grain boundaries with a low as we11 as a gradually increasing concentration of impurities are then presented. The relationship between the boundary structure and the propensity to segregation together with the structural changes invoked by segregation, are discussed in the light of these calculations.
}

\section{Introduction}

Segregation of solutes and/or impurities to grain boundaries is a general phenomenon which occurs both in metalic and non-metallic materials an it may affect significantly various material properties. For example, increased concentration of alloying elements at grain boundaries leads to changes in grain boundary diffusivity [1] and it also influences the ability of boundaries to act as sources and sinks of vacancies [2]. This, in turn, affects, for example, the creep properties of materials, in particular the creep fracture [3]. Similarly, migration of grain boundaries is to a large extend controlled by their interaction with impurities [4]. The presence of impurities at grain boundaries also invokes local changes in the electronic structure of the material and often leads toia significant decrease of cohesion at the interface. The former may have a pronounced effect, for example, on the electronic properties of semiconductors and the latter increases the susceptibility to intergranular fracture [5-7]. 
The thermodynamical, phenomenological description of segregation has been developed by a number of authors (e.g. [8-10]). In this context the propensity for segregation has usually been interpreted in terms of general physical and chemical characteristics of the alloys and their components such as differences in atomic radii and electronegativities or solid solubility. However, the discrete atomistic structure of grain boundaries has not been considered in these developments. On the other hand, there is an ample experimental evidence that the structure of grain boundaries plays a role in the segregation process. The segregation has been observed to be anisotropic and selective [11-14] as we11 as dependent upon the misorientation of the grains [15] and orientation of the boundary plane [16]. The promotion of faceting by segregation [17-19] and the observed changes in the number of low energy boundaries detected in the sintering of spheres experiments [20] suggest that segregation induces significant changes in the grain boundary structure. A detailed review of these phenomena can be found in [21]. Hence, atomistic studies of grain boundaries with segregated impurities are needed to incorporate the structural aspects into the theoretical description of segregation. The first aim of such studies must be to investigate the role the large variability of grain boundary structures plays and to assess the relative importance of the structural and chemical factors for the propensity to segregation. Understanding of possible structural changes in the boundaries invoked by the segregation, their relationship to chemical changes and the influence of both upon the grain boundary phenomena is another goal of the atomistic studies.

Until now only very limited atomistic studies of grain boundaries with impurities have been performed, usually for low concentrations of the segregant. All the calculations which considered fully the grain boundary structure and possible relaxations due to the impurities, have been made using empirical central forces to describe the atomic interactions. On the other hand possible changes in the cohession invoked by the presence of impurities have been discussed recently using the molecular orbital cluster methods $[22,23]$ and by describing the bonding using an analogy with the theoretical description of chemisorption $[24,25]$. In both cases it has been concluded that the embrittling elements may cause a decrease in bonding of neighbouring metal atoms owing to the formation of covalent bonds between the impurities and the host atoms. However, since no features of the atomic configurations specific to the grain boundaries have been included into those studies these conclusions are of general nature, independent on whether the impurity is in the boundary or elsewhere.

The first atomistic calculations with full relaxation which employed the central forces have been made by Weins and Weins [26] and by Beeler and coworkers $[27,28]$. In the former case $C u$ was studies as a substitutional impurity in Au and a significant anisotropy of segregation was found. In the latter case the investigation of carbon as an interstitial impurity in f.c.c. iron has been made and it was found that in the vicinity of $C$ atoms more close packed atomic configurations have been formed. A similar study has also been made by Nichols [29]. More recently an atomistic study of grain boundaries with impurities was performed by Machlin and Levi [30] for a model f.c.c. system employing the potentials constructed in ref. [31]. They concluded that segregation was favoured when the atomic diameter of the solute was larger than that of the host and also when the cohesive energy of the solute was smaller than that of the host. A Monte-Carlo simulation of the equilibrium distribution of impurities in the vicinity of two $\Sigma=5$ grain boundaries has been performed in ref. [32] using a Morse potential for the Cu-Bi system. A good agreement with the Mclean's isotherm [8] was found. The Morse potential has also been employed in the recent study [33] of the $\Sigma=5$ boundary in the b.c.c iron with phosphorus as an impurity. In this study both the dilute and high concentrations of $P$ have been considered and in the latter case a transformation of the boundary structure was observed.

An empirical description of interatomic forces in alloys has recently been developed in ref. [34] with the afm to use it in the atomistic studies of crystal defects. In this scheme the energy of the system is expressed in terms of pair potentials and a density dependent term. This description of interatomic forces has then been employed in a systematic study of boundaries containing low concen- 
tration of impurities for the systems $\mathrm{Cu}-\mathrm{Bi}, \mathrm{Cu}-\mathrm{Ag}$ and $\mathrm{Au}-\mathrm{Ag}$ [35]. In the present paper we first summarize the main aspects of the grain boundary structure which need to be considered when studying segregation. We then outline briefly the above mentioned empirical scheme of the description of interatomic forces and discuss the general, structure independent, properties of segregation which can be deduced in the framework of this scheme. Finally, we discuss the atomistic aspects of segregation, first for the case of low concentration of the solute in the boundary when no direct interactions between individual solute atoms exist, and then for the gradually increasing concentration. This we demonstrate on the example of the $\mathrm{Cu}-\mathrm{Bi}$ system and the $\Sigma=5$ (310) tilt boundary.

\section{Atomic Structure of Grain Boundaries}

The majority of atomistic calculations have been carried out for high coincidence, low $\Sigma$, boundaries. Tilt boundaries have been analysed more frequently than twist boundaries and no study of mixed tilt and twist boundaries has been made. In general, it has been found that the boundary region is very narrow, of the order of one to two lattice parameters and the atomic configurations in the boundaries may often be described as compact polyhedra [36-38] which are analogous to the polyhedra identified by Bernal [39] in models of liquids but, in general, they are not close-packed. An important relaxation at the grain boundaries is the relative translation of the grains away from the coincidence position $[38,40,41]$ so that there are then no coincidence atoms at the boundary. However, the question arises whether these results are specific for the low $\Sigma$ boundaries or equally applicable to general boundaries and if so what is the relationship between the high coincidence and genera1 boundaries.

Any general boundary can be approximated as closely as required by a high $\Sigma$ coincidence boundary. When this is done the use of coincidence allows us to describe the general boundary as periodic but it has no other physical significance. Hence, structures of high $\Sigma$ symmetrical and asymmetrical tilt boundaries have been investigated [42-44] in order to clarify the atomic structure of general boundaries. The most important conclusion of these studies is that for a given inclination of the boundary plane, all high $\Sigma$ boundaries, in a certain misorientation range, can be described as composed of two types of units. The dimensions of these units are such that the boundaries that delimit this misorientation range may each be constructed as a contiguous sequence of one type of these units. Indeed, a contiguous sequence of, at least one of these units, always corresponds to a mechanically stable structure of one of the delimiting boundaries. This boundary is called favoured. If contiguous sequences of both types of units, which is usually the case, lead to stable structures then any general boundary, also called non-favoured, in the misorientation range delimited by the two favoured boundaries, is composed of a unique sequence of units of these two boundaries. (When the translation states of the delimiting boundaries are not compatible units of intrinsically unstable structure may be introduced into the non-favoured boundaries [44]). It has recently been shown that this description of high $\Sigma$ boundaries applies also to the twist boundaries ([45], D. Schwartz, A. P. Sutton and V. Vitek, to be published) and it is likely to be entirely general, applicable also to mixed boundaries. Examples of two favoured boundaries, $\Sigma=5(210)$ and $\Sigma=5(310)$, calculated using a potential for $\mathrm{Cu}$ [34], are shown in Figs 1 and 2, respectively. An example of a non-favoured boundary is shown in Fig. 3 where the period of the $\Sigma=73$ (830) boundary is seen to be composed of the two units of the (310) boundary and of the one unit of the (210) boundary.

In the atomistic studies of grain boundaries it has been frequently found that more than one stable structure may exist for a given geometrically defined boundary [38]. Some of these structures may be symmetry related [41] while others are not and possess different energies. Our recent studies (Gui Jin Wang, A. P. Sutton and $\mathrm{V}$. Vitek, to be published) have shown that the multiplicity of grain boundary structures is a very general phenomenon and in the case of general boundaries a very large number of different structures which possess very similar energies, may exist for any geometrically well defined boundary. The reason is that several dif- 


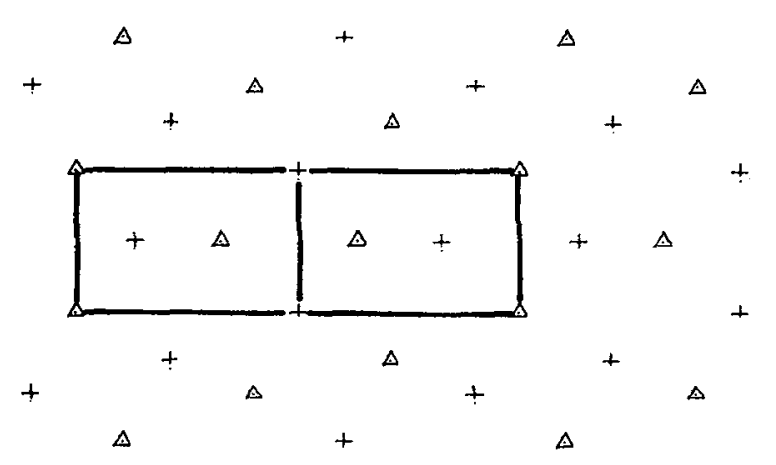

Fig. 1. $\Sigma=5(210)$, $\left.36.87^{\circ}\right][001]$ symmetrical tilt boundary in $\mathrm{Cu}$ (structure $\mathrm{B}$ ). + and $\Delta$ represent here and in all the following pictures, atoms in different (002) planes. Two units of the boundary are marked by full lines.

$\Delta$

$\Delta$

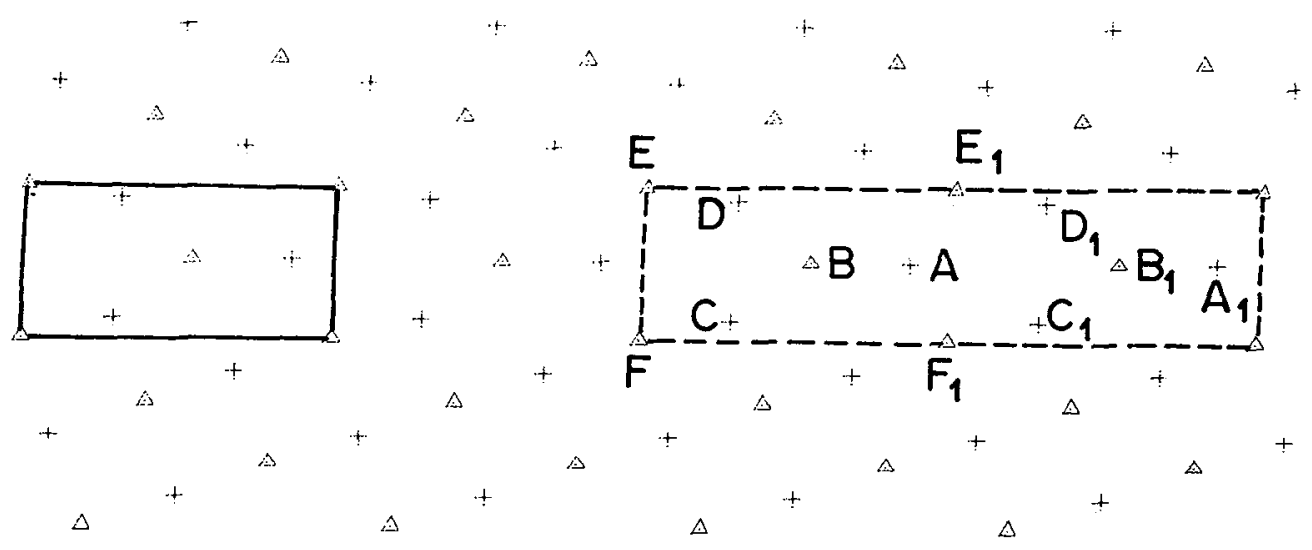

Fig. 2. $\Sigma=5(310), 53.13^{\circ} /[001]$ symmetrical tilt boundary in Cu. The basic unit of the boundary is marked by full lines. The unit cell used in the studies of this boundary with solutes is marked by dashed lines.

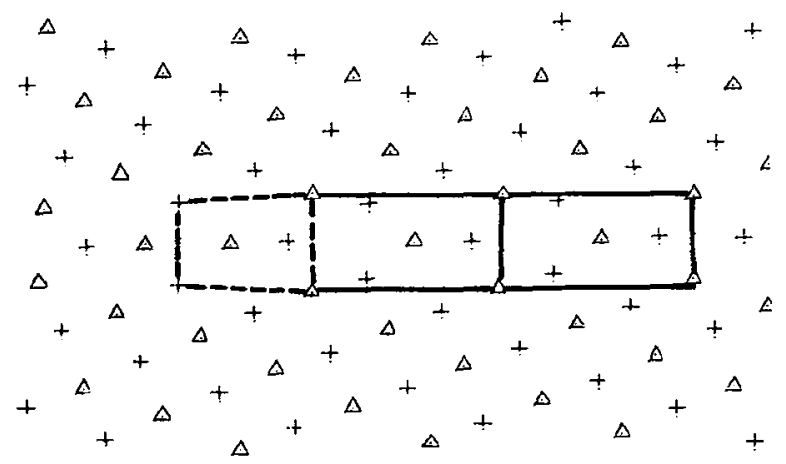

Fig. 3. $\Sigma=73(830)$, $48.39^{\circ} \%[001]$ symmetrical tilt boundary in $\mathrm{Cu}$. The units of the (310) boundary are marked by full lines and the units of the (210) boundary by dashed lines. 
ferent structures of favoured boundaries may occur which can then all play the role of structural units in the general boundaries. For example, if the two favoured boundaries, delimiting a certain misorientation range, occur in $i$ and $j$ different configurations, respectively, a non-favoured boundary composed of $n$ units of the first and $m$ units of the second boundary may exist in $i^{n} \cdot j^{m}$ different configurations; not all these conflgurations are of course, necessarily stable or of low energy. This large multiplicity of grain boundary structure may be instrumental in explanation of the ease of diffusion, ability of boundaries to act as sinks and sources for vacances etc. (Gui Jin Wang, A. P. Sutton and V. Vitek, to be pub1ished).

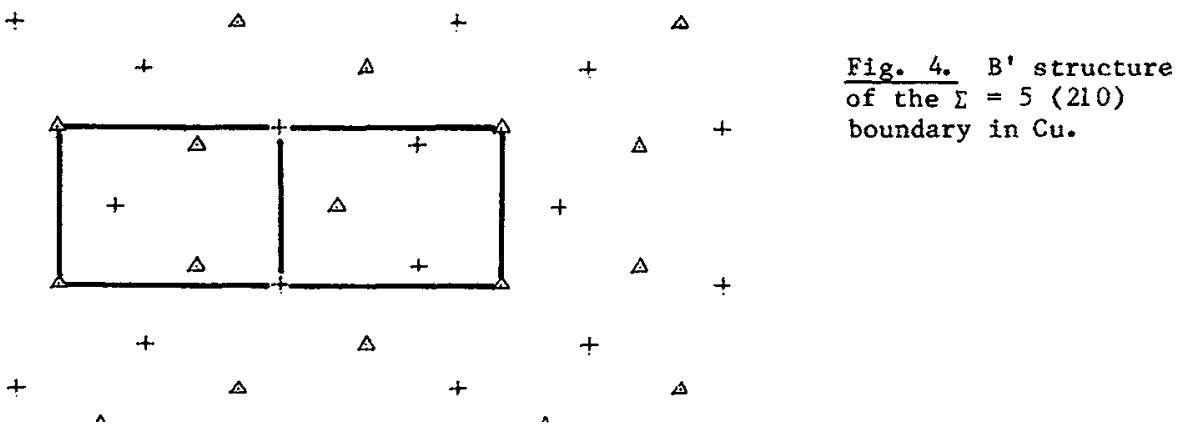

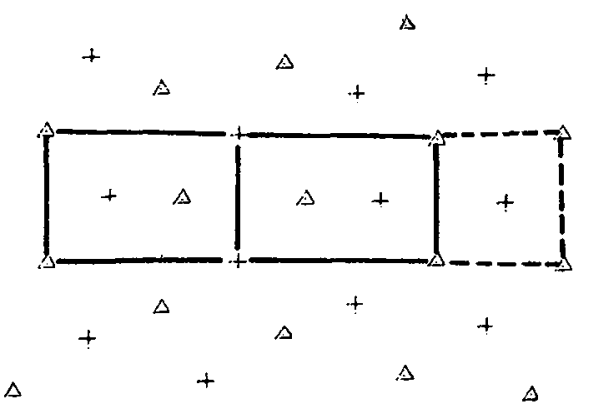

(a)

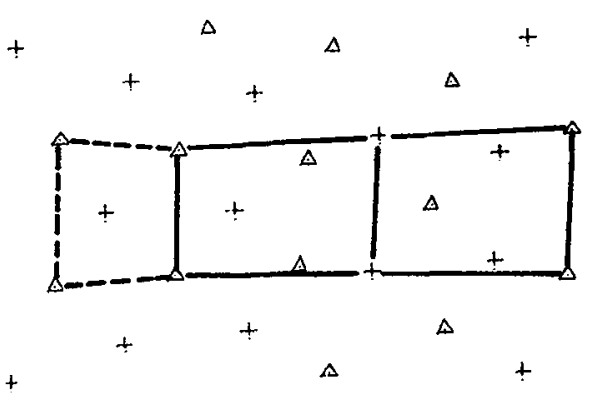

(b)

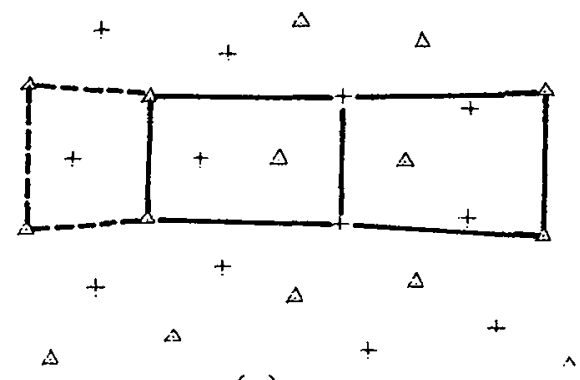

Fig. 5. $\sum=17(530), 28.07^{\circ} /[001]$ symetrical tilt boundary in Cu. (a) $A B B$ structure, (b) $A B^{\prime} B^{\prime}$ structure, (c) $A B B$ ' structure. Units of the (210) boundary are marked by full lines and units of the ideal crystal by dashed lines.

(c) 
As an example another configuration of the favoured $\Sigma=5$ (210) boundary is shown in Fig. 4. The ratio of the energy of this structure to that shown in Fig. 1 is 1.04. Consider now, for example, $\Sigma=17$ (530) boundary which is composed of two units of the $(210)$ boundary and one unit of the ideal crystal $(\Sigma=1(110))$. Using the two available (210) structures this boundary may exist in four modifications. If we mark units of the ideal crystal $A$ and units of the boundaries shown in Figs. 1 and $4 B_{\text {and }} B^{\prime}$, respectively, these are $A B B, A B^{\prime} B^{\prime}, A B B^{\prime}$ and $A B^{\prime} B$. The first three of these configurations have, indeed, been found to be stable and of low energy. They are shown in Fig. 5 and the ratios of their energies are $1.07: 1.09: 1.0$.

This model of the grain boundary structure suggests that properties of low $\Sigma$, short period, boundaries may be extrapolated with good confidence to high $\Sigma$ boundaries and thus to the boundaries of general type. It also implies that the properties controlled by the core of the boundaries, e.g. propensity to segregation, although not the same for all boundaries, need not show any extreme behaviour at favoured boundaries. However, a discontinuous change in the derivative of these properties with respect to misorientation may occur at a favoured boundary since different structural elements are introduced at higher and lower misorientations than the favoured boundary misorientation [44]. It is possible, however, that some of the favoured boundaries, such as $\Sigma=3$ (111) twin, possess particularly "ordered" structures and thus particularly low energy, propensity to segregation etc. These boundaries could then be termed special. The multiplicity of grain boundary structures suggest that a possible effect of segregation may be occurance of transformations between different structure and/or enhancement of the occurance of one or several of the large number of structures available in the case of general boundaries. These phenomena can, however, be only assessed when carrying atomistic studies of boundaries with impurities.

\section{Description of interatomic forces and general aspect of segregation.}

In the empirical description of interatomic forces which has been developed in ref. [34], the energy per atom $i, E_{i}$, in a binary alloy $A_{1-X^{B} X}$ is

$$
E_{i}=\frac{1}{2} \sum_{\substack{j=1 \\ j \neq i}}^{N} \phi^{i j}\left(r_{i j}\right)+U_{v}\left(v_{a}\right)
$$

where $\mathrm{N}$ is the total number of atoms and $\Phi^{i j}$ is, depending on the species of atoms $i$ and $j$, either $\Phi^{A A}, \Phi^{B B}$ or $\Phi^{A B}$ which are the three potentials describing $A-A, B-B$ and $A-B$ interactions, respectively. $U_{v}$ is the density dependent part of the energy which is a function of the average volume per atom and it has been taken as

$$
U_{v}=(1-X) U_{v}^{A}+X U_{v}^{B}
$$

where $U_{v}^{A}$ and $U_{v}^{B}$ are the corresponding density dependent parts of the energy for the pure elements $A$ and $B$. $U Q d(\alpha=A, B)$ is further written in the form of an expansion:

$$
\mathrm{u}_{\mathrm{v}}^{\alpha}=\mathrm{u}_{\mathrm{v}}^{\alpha}\left(\mathrm{v}_{\mathrm{a}}^{\alpha \circ}\right)+\mathrm{u}_{\mathrm{v}}^{\alpha^{\prime}}\left(\mathrm{v}_{\mathrm{a}}^{\alpha o}\right)\left(\mathrm{v}_{\mathrm{a}}-\mathrm{v}_{\mathrm{a}}^{\alpha o}\right)+\frac{1}{2} \mathrm{u}_{\mathrm{v}}^{\alpha^{\prime}}\left(\mathrm{v}_{\mathrm{a}}^{\alpha \circ}\right)\left(\mathrm{v}_{\mathrm{a}}-\mathrm{v}_{\mathrm{a}}^{\alpha o}\right)^{2}
$$

where $v_{a} \alpha^{\circ}$ is the atomic volume of the pure element $\alpha$ in equilibrium. 
The potentials $\phi^{i j}$ were represented by third order polynominals together with a repulsive term proportional to $r^{-4}$. For pure metals the parameters in the potentials and volume dependent terms were chosen such as to fit the cohesive energy and elastic constants of the material (note that Cauchy relations are not satisfied in this case) and to satisfy the conditions of mechanical stability for a given structure and lattice parameter; resonable values of the stacking fault energy have also been required in the case of f.c.c. and hexagonal crystals. For alloys the possible charge transfer was first taken into account following the procedure suggested by Machlin [31], by introducing reference structures of $A$ and $B$ elements with modified lattice parameters and adjusting the repulsive parts of $A-A$ and $B-B$ potentials. The $A-B$ potential was then determined by requiring that the lattice of the random substitutional alloy be mechanically stable for the lattice parameter determined using a modified Vegard's law and by fitting self-consistently the enthalpy of mixing. The latter means that for low concentrations $(X \leqslant 0.05)$ the enthalpy of mixing was fitted when taking fully into account the relaxation of atoms $A$ in the vicinity of a substitutional atom $B$. The effects of this relaxation were then extrapolated to higher concentrations but full relaxation calculations had not been made for higher values of $X$. These procedures, together with the discussion of the validity of the corresponding potentials, have been discribed in detail in ref. [34].

When studying grain boundaries we always assume that their structure is periodic. In the case of boundaries with impurities the corresponding repeat cell may be a multiple of the repeat cell of the pure boundary, depending on the concentration of the solute in the boundary. Let us assume that there are $N=N_{A}+N_{B}$ atoms in the repeat cell of a boundary, where $\mathrm{N}_{A}$ is the number of solvent atoms and $N_{B}$ the number of solute atoms. Assuming tha $N_{A}>N_{B}$ the energy per unit area of this boundary, measured with respect to the ideal crystal of the solvent, is

$$
\begin{aligned}
& \gamma_{b}^{N} N_{B}^{N}=\frac{1}{S p}\left[\frac{1}{2} \sum_{i=1 j}^{N} \sum_{1 j}^{i j}\left(r_{i j}\right)+N_{A} U_{\dot{v}}^{A}\left(v_{a}^{A o}\right)+\right. \\
& \left.+\mathrm{U}_{\mathrm{v}}^{\mathrm{A}^{-}}\left(\mathrm{v}_{\mathrm{a}}^{\mathrm{Ao}}\right) \Delta \mathrm{v}^{\mathrm{N}} \mathrm{B}+\sum_{\mathrm{k}=1}^{\mathrm{N}}{ }^{\mathrm{B}} \mathrm{U}_{v}^{\mathrm{B}}\left(\mathrm{v}_{\mathrm{ak}}^{\mathrm{B}}\right)-\mathrm{NE}_{\mathrm{c}}^{\mathrm{A}}\right]
\end{aligned}
$$

where $S_{p}$ is the area of the repeat cell in the boundary plane, $\Delta V$ is the total volume change per one repeat cell of the boundary for this concentration of the solute, $v_{a k}^{B}$ is the atomic volume of the solute $k$ in the boundary (evaluated as described in [35]) and $\mathrm{E}_{\mathrm{c}}^{\mathrm{A}}$ is the cohesive energy per atom of the solvent. Summation over $i$ extends over all atoms in the ceil and summation over $j$ extends over all atoms interacting with atom $i$. When another solvent atom is replaced by the solute atom the energy of the boundary changes to $\gamma_{\mathrm{b}}^{N}{ }^{-1},{ }_{B}+f^{+i v e n}$ by equation (4) in which $\mathrm{N}_{\mathrm{A}}$ is replace by $\mathrm{N}_{\mathrm{A}}^{-1}, \mathrm{~N}_{\mathrm{B}}$ by $\mathrm{N}_{\mathrm{B}}^{+1}$ and $\Delta \mathrm{V}_{\mathrm{B}}$ by $\Delta \mathrm{V}^{\mathrm{N}_{\mathrm{B}}+1}$; note that the contributions of the pair potential terms are also different in the two cases. The change of the boundary energy per one solute atom is then

$$
\Delta \gamma_{B}^{N}=s_{p}\left(\gamma_{b}^{N} A^{-1}, N_{B}+1-\gamma_{b}^{N}{ }^{N} N_{B}\right)
$$

and the enthalpy of mixing corresponding to placing the solute into the boundary which originally contained $N_{B}$ solute atoms in the unit cell, is

$$
\Delta \mathrm{H}_{\mathrm{m}}^{\mathrm{N}_{\mathrm{B}}}=\Delta \gamma_{\mathrm{b}}^{\mathrm{N}}+\Delta \mathrm{E}_{\mathrm{c}}^{\mathrm{AB}}
$$


where

$$
\Delta E_{c}^{A B}=E_{c}^{A}-E_{c}^{B}
$$

is the difference between the cohesive energies of the solvent and the solute.

The energy of segregation, ${ }_{\mathrm{S}}^{\mathrm{N}} \mathrm{B}$, corresponds to the change of the energy of the system when a solvent atom in the boundary containing already $\mathrm{N}_{B}$ solute atoms in the unit cell, is replaced by a solute which was originally in the bulk. It is

$$
\mathrm{E}_{\mathrm{s}}^{\mathrm{N}}=\Delta \underset{\mathrm{mb}}{\mathrm{N}_{\mathrm{B}}}-\Delta \mathrm{H}_{\mathrm{m}}^{\mathrm{B}}
$$

where $\Delta \mathrm{H}_{\mathrm{m}}^{\mathrm{B}}$ is the enthalpy of mixing per one solute atom in the ideal lattice of the solvent. The condition for segregation, $\mathrm{E}_{\mathbf{s}} \mathrm{B}<0$, may then be written as

$$
\Delta \gamma_{b}^{N_{B}}<\Delta H_{m}^{B}-\Delta E_{c}^{A B}
$$

$\Delta \mathrm{H}_{\mathrm{m}}^{B}$ and $\Delta \mathrm{E}_{\mathrm{c}}^{\mathrm{AB}}$ are both constants for a given alloy system. Hence, the upper limit of the change of the grain boundary energy associated with introduction of a solute to the boundary, for which segregation is favoured, is independent of the grain boundaxy structure. For the systems considered in [35] and in the next section the right side of the inequality (9) is always positive (for $\mathrm{Cu}-\mathrm{Bi}$ system it is $1.799 \mathrm{eV}$ ) and thus segregation may be favourable even though the boundary energy increases.

In contrast with the right side of (9), $\Delta \gamma_{b}{ }^{N}$ is strongly dependent on the atomic environment of the boundary site considered (see next section) and for a given alloy system it can lie within a broad band of values. In a polycrystal the number of boundaries is very large and the distribution of misorientations is random. Hence many possible sites exist and it is therefore likely that the inequality (9) can always be satisfied, at least initially, at some boundary sites. Thus at least a limited segregation is likely to occur in any alloy system. As discussed in more detail in [35], the probality that segregation occurs when a large number of different segregation sites are avallable, is the higher the larger is the allowed change in the boundary energy, i.e. the larger is $\Delta \mathrm{H}_{\mathrm{m}}-\Delta \mathrm{E}_{\mathrm{c}} \mathrm{B}$. This suggests first that the prepensity for segregation should increase as $\Delta H_{m}^{B}$ increases i.e. as the solubility decreases. This is generally in good agreement with the experimental observations $[9,46]$. Secondly, the propensity for segregation should increase as $\triangle \mathrm{E}_{\mathrm{C}}^{\mathrm{AB}}$ decreases, i.e. the lower is the cohesive energy of the solute and the higher is the cohesive energy of the solvent. This has also been found in calculations of Machlin and Levi [30].

It should be emphasized that the above dependence of the propensity to segregation on $\Delta H_{m}^{B}$ and $\Delta E_{c}^{A B}$ applies only in average and cannot be applied to any specific segregation site where the effect of the local environment can override these general trends. These aspects of segregation as well as any changes in grain boundaries invoked by segregation can only be investigated using atomistic models of grain boundaries.

\section{Atomistic studies of grain boundaries with impurities}

Atomistic calculations of the $\Sigma=5$ (210), structure $B$ (Fig. 1) and $\Sigma=17$ (530), structure $\mathrm{ABB}$ (Fig. 5a) tilt boundaries in Cu and $\mathrm{Au}$ with $\mathrm{Bi}$ and $\mathrm{Ag}$ 
as impurities, have been made in ref. [35] for low concentrations of the solutes in the boundaries. Recently, similar calculations have also been made for $\sum=5$ (210) structure $B^{\prime}$, for the other two $\Sigma=17$ (530) structures ( $A B^{\prime} B^{\prime}$ and $A B B^{\prime}$ ) and for the $\Sigma=5(310)$ boundary shown in Fig. 2 (Gui Jin Wang and.V. Vitek, to be published). In all these calculations solutes were introduced into the grain boundaries periodically in rectangular lattices with sides parallel and perpendicular to the tilt axis. For the $\Sigma=5$ (310) boundary this lattice has sides [1 $\overline{3} 0]$ and [002], respectively. The spacing of the impurities then corresponded to concentrations $0.096,0.074$ and 0.068 of a monolayer for the (210), (530) and (310) boundaries, respectively. (One monolayer would correspond to the close-packed layer of solutes assuming the lattice parameter of the solvent). For these concentrations the spacing of the solutes was always sufficiently large that no impurity interacted with another directly. Impurities were introduced successively to a number of substitutional, non-equivalent sites, such as sites $A-F$ marked in Fig. 2 . The full relaxation calculation which allows for the relative displacements of the grains, local readjustment of atom positions and also for an expansion perpendicular to the boundary, was carried out in each case employing the calculation method described in detail in ref. [35]. Since the solutes have been introduced perlodically into the boundaries no overall extension parallel to the boundary plane can take place. This represents a constraint imposed on the relaxation but if a net area change were allowed parallel to the boundary it would accumulate into a fintte strain which would produce long range strain fields in the adjoining grains. This strain could be relieved, of course, by the formation of a network of grain boundary dislocations which indeed occurs in the transition from coherent to semicoherent interfaces. However, in our case, owing to the low concentrations of impurities, we are far removed from this situation and the above mentioned long range field would only lead to a substantial energy increase. This will not occur, however, if the periodicity is malntained and an overall volume change occurs only by an expansion or contraction normal to the boundary.

At low concentrations the main characteristics of the propensity to segregation as well as the effect of the solutes upon the grain boundary structure was found to be similar in all boundaries studied. The most significant structural effect on the propensity to segregation is the anisotropy of segregation sites that is manifested by the very significant variation in segregation energies from one atomic site to another. This is particularly pronounced in the Cu-Bi system. An example is shown in Table 1 where $E_{S}$ is listed for the case of $B i$ at different sites in the $\tilde{\Sigma}=5(310)$ boundary in $\mathrm{Cu}$. (Note that the sites $\mathrm{D}$ and $\mathrm{C}$ are not equivalent because of the lack of mirror symmetry across the boundary plane which is caused by the relative displacement of the top grain with respect to the bottom grain by $0.04[1 \overline{30}]$ ).

TABLE $\quad 1$

\begin{tabular}{lllllll} 
Site & A & B & C & D & E & F \\
\hline $\mathrm{E}_{\mathrm{S}}(\mathrm{eV})$ & 0.80 & -2.0 & 0.35 & 0.7 & -2.0 & -1.8
\end{tabular}

Fig. 6 shows the hydrostatic stress field of this boundary and comparison with the Table 1 shows that the segregation energy is positive for the sites associated with compression and negative for these sites which are associated with tension. This suggests that it is the size effect that governs the propensity for segregation of B1 to grain boundaries in Cu. An analogous behaviour has been observed in the case of $\mathrm{Ag}$ as an impurity in $\mathrm{Cu}$ [35]. In this case positive segregation energies were found for all the sites studied but they were lower for the tensile sites. However, favoured segregation was found for the $\Sigma=17$ (530) boundary with $A B^{\prime} B^{\prime}$ structure as well. as for the $\Sigma=5(310)$ boundary (Gui Jin Wang and V. Vitek, to be published). In the case of $\mathrm{Ag}$ as an impurity in $\mathrm{Au}$ no significant differences in the segregation energy exist between tensile and compressive sites (Ag and Au atoms possess almost the same atomic volumes) and segregation was found to be favoured for all the sites studied [35]. 
The detailed analysis of the relaxed structures indicates that although no drastic changes in the boundary structure occur, significant local relaxation always takes place around solute atoms. These relaxations differ for each case and they cannot be related only to the types of solutes and solvents but depend on the local structure of the boundary. They ultimately decide whether the segregation is favoured at a grain boundary site, since they deterimine whether it is possible to relax the atomic configuration around an impurity atom so as to produce the optimum atomic environment for the impurity atom without excessively distorting the host boundary structure. In the case of $\mathrm{Bi}$ in $\mathrm{Cu}$ such sites are usually those associated with a local expansion but no such identification can be made in the case of $\mathrm{Ag}$ in $\mathrm{Au}$.

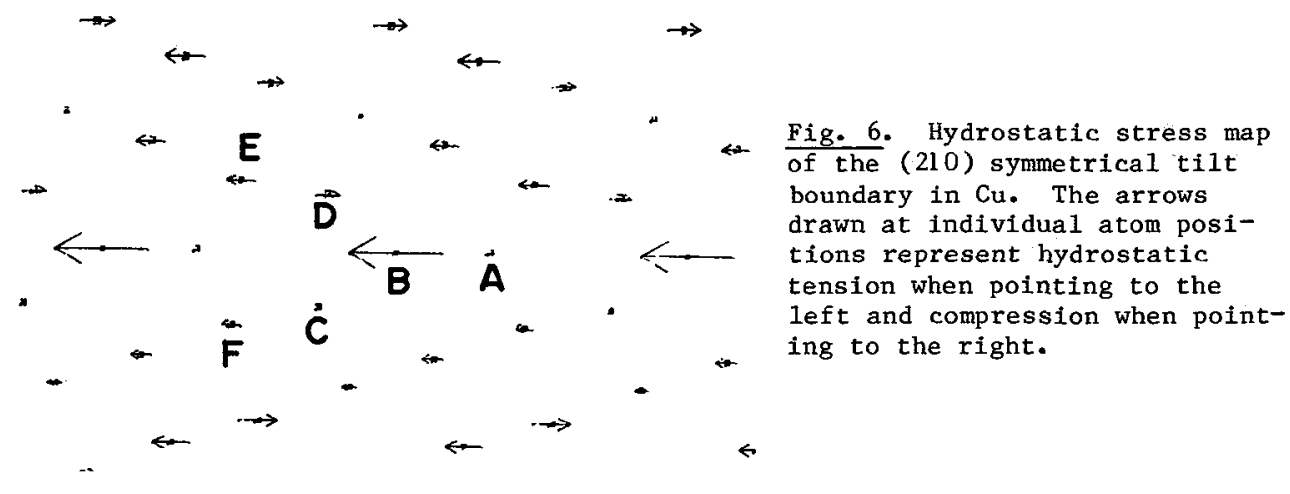

No substantial differences in both the propensity to segregation and the concommitant changes in the boundary structure were found between the (210) and (310) favoured boundaries and the non-favoured (530) boundary. Furthermore, similarities between the behaviour of the sites in the (210) boundary and the corresponding sites in the (210) units of the (530) boundary were observed. Thus no unusual features of the segregation have been observed for the favoured boundaries (210) and (310) when compared with the non-favoured (530) boundaries which is in agreement with the general discussion in section. 2. On the other hand the energy of each of the three different structures of the $\Sigma=17$ (530) boundary changes differently with segregation. For example for the case of $\mathrm{Cu}$ when $\mathrm{Bi}$ is placed to the most favourable sites in each of these structures the ratios of their energies in the order $A B B: A B^{\prime} B^{\prime}: A B^{\prime} B$ are 1.18:1.06:1.0. Comparing with the ratios given in section 2 for the pure boundaries it is seen that the energy of the $A B B$ structure increases substantially while the energies of the $A B^{\prime} B^{\prime}$ one $A B B^{\prime}$ structures move closer together. Hence, segregation may eliminate some of the possible structures of general boundaries while making others more equivalent.

In order to study the dependence of the propensity to segregation upon the solute concentration and solute-solute interaction as well as to investigate possible structural changes associated with increasing concentration of the solute at boundaries, atomistic studies of grain boundaries with much higher content of impurities need to be carried out. These studies are presently in progress and here we report only some preliminary results of the calculations of the $\Sigma=5$ (310) boundary in $\mathrm{Cu}$ with gradually increasing amount of segregated $\mathrm{Bi}$. As in the calculations for low concentrations of impurities the periodicity of the structure is assumed throughout and the repeat cell is always [130]x[002]. Assuming that the first $B i$ atom segregated into the $B$ site (large negative segregation energy) the second $B i$ atom was put successively into a number of sites listed in Table 2 (see also Fig. 2) where the corresponding segregation energies are also given. 
TABLE 2

$\begin{array}{llllllll}\text { Site } & \mathrm{B}_{1} & \mathrm{C} & \mathrm{C}_{1} & \mathrm{E} & \mathrm{E}_{1} & \mathrm{~F} & \mathrm{~F}_{1} \\ \mathrm{E}_{\mathrm{S}}(\mathrm{eV}) & -1.8 & 0.7 & -1.0 & -2.0 & -2.1 & -1.7 & -1.8\end{array}$

It is seen that the presence of the Bi atom at the B site affects only slightly the segregation energies for the sites $B_{1}, E, E_{1}, F$ and $\left.F\right\rfloor$ ( $B 1, E I, F l$ are equivalent to $B, E, F$, respectively in the pure case). On the other hand $C_{1}$ site now becomes favourable for segregation while the segregation energy for the site $C$ (and $C_{1}$ ) is positive when no other atom is present in the unit cell (see Table 1). This is obviously a consequence of the Bi-Bi interaction which is still negligible when $B i$ is at the sites $B$ and, for example, at $E_{I}$ but becomes important and strongly attractive when it is at the site $B$ and $C_{1}$. When two $B i$ atoms are present in the unit cell of the boundary the relaxation is generally more extensive and the relative displacement of the two grains away from the coincidence position increases.

When two sites with the lowest segregation energies, e.g. $B$ and $B_{1}$ or $B$ and $E_{1}$ are occupied by $B i$ atoms then the third $B i$ atom can be most favourably placed into the sites $E_{1}$ or $B_{1}$, respectively, since they correspond to the lowest segregation energies in this case. The fourth Bi atom is then most favourably placed into the site $\mathrm{E}$ but other sites become also available, for instance site A possesses now a negative segregation energy, although it was unfavourable for segregation originally. Thus the number of sites favourable for segregation increases with encreasing concentration of $\mathrm{Bi}$. The structure of the $\Sigma=5$ (310) boundary containing $B i$ atoms at the sites $B, B_{l}, E$ and $E_{l}$ is shown in Fig. 7. Clearly the structure has now changed significantly when compared with that of the pure boundary. The most pronounced is the increase of the relative displacement of the upper grain with respect to the lower grain away from the coincidence position; this displacement is now 0.08 [ $1 \overline{3} 0]$. The configuration formed when $\mathrm{Cu}$ atoms were successively replaced by $\mathrm{Bi}$ atoms at those sites which possessed the lowest segregation energy, appears to be a two-dimensional ordered structure in which every Bi atom is surrounded by $\mathrm{Cu}$ atoms and vice versa. This is, of course, enabled by the periodicity of the grain boundary structure. The basic features of this ordered structure, such as the average separation of $\mathrm{Bi}$ atoms, appear to be determined by the $\mathrm{Bi}-\mathrm{Bi}$ and $\mathrm{Cu}-\mathrm{Bi}$ interactions; for example, in the structure shown in Fig 7 the Bi-Bi separations closely coincide with the distance at which the Bi-Bi potential has a minimum. On the other hand the details of this ordered layer are related to the original atomic structure of the grain boundary. Hence, if such ordered layers are formed at different boundaries it is likely that the average separation of solutes and solvents in them will be similar while details of these configurations will depend on the structure of the given boundary. It is, of course, possible

$\Delta$

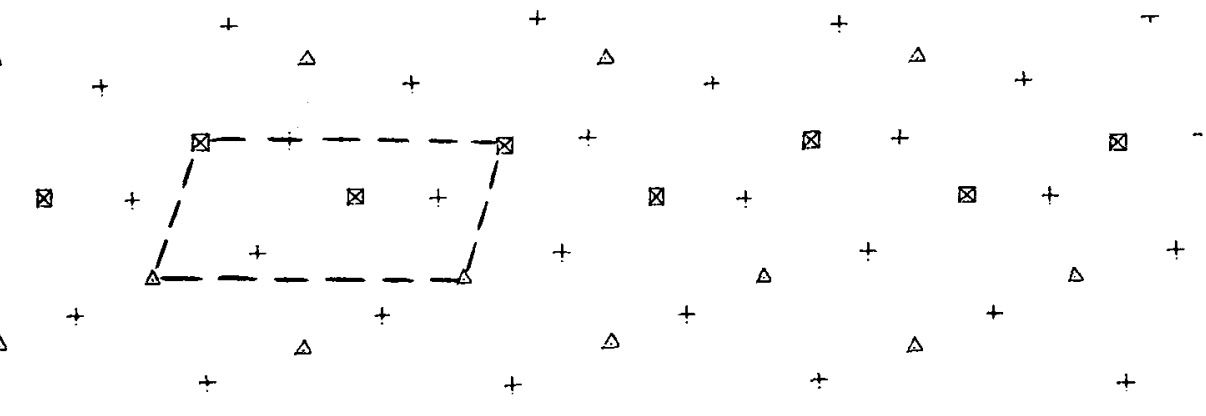

Fig. 7. $\Sigma=5(310)$ symmetrical tilt boundary in $\mathrm{Cu}$ with $\mathrm{Bi}$ atoms (depicted as (4) segregated to the $B$ and $E$ sites in each period. The original unit of the boundary is marked by dashed lines. 
that further decrease of the energy of the system may be achieved by removing the solvent atoms from these structures without replacing them by solute which then leads to formation of a layer of the precipitated solute at the boundary.

\section{Conclusions}

The atomic structure of general boundaries can always be regarded as composed of two types of units at least one of which corresponds to the unit cell of a stable, short period boundary which we call favoured. Hence, complete continuity of segregation behaviour is expected in large ranges of misorientations. No extreme behaviour is likely to occur at the majority of favoured boundaries but several special cases may exist. In general, multiplicity of structures exists for a given geometrically defined boundary. This multiplicity can be very extensive in the case of general boundaries which may be important for their physical properties.

From the calculation of the segregation energy within the proposed scheme of the description of atomic interactions it follows that segregation is favoured when the grain boundary energy (per one solute atom) changes upon segregation by less than $\Delta \mathrm{H}_{\mathrm{B}}^{\mathrm{B}}-\Delta \mathrm{E}_{\mathrm{C}}^{\mathrm{AB}}$. This suggests that in polycrystals where the variability of the types of boundaries is large, the segregation propensity is likely to increase with increasing enthalpy of mixing and decreasing cohesive energy of the solute. This rule cannot, however, be applied to individual segregation sites. At this level the segregation is very anisotropic and the only criterion for segregation is whether a favourable atomic environment for the impurity atom can be found. This implies that no rule for segregation propensity to any particular boundary site can be established, that is based only on physical and chemical characteristics of the alloy and its components. Thus a level of segregation is likely to occur in any binary system and the question is then whether the concentration of the solute will grow on these boundaries where segregation was initiated. This appears to be mainly controlled by the type of the solute-solute and solute-solvent interactions and, therefore, the physical and chemical properties of the solutes and solvent may govern this part of the segregation process. Since the grain boundaries are, at least locally, always periodic two dimensional ordered phases may form at the grain boundaries as the concentration of the segregant increases. The average properties of these phases will be governed by the atomic interactions but the details will depend on the original atomic structure of each boundary. At the same time significant changes in the grain boundary structure occur. For example, relative displacements of the grains both parallel and perpendicular to the boundary are altered; the latter corresponds to the expansion or contraction at the boundary. Since these displacements can, in some cases, be detected experimentally using the transmission electron microscopy [41,47], their measurements could provide a technique for experimental investigation of the atomistic effects of segregation. In the case of general boundaries segregation also affects the multiplicity of structures which may then be reflected in various physical properties of the boundaries.

Acknowledgements - This research has been supported by the United States Department of Energy, Contract No. DE-ACO2-79ERI-0429.

\section{References}

[1] Peterson N. L., Grain Boundary Structure and Kinetics, American Society for Metals (1980) p. 209.

[2] Balluffi R. W., Grain Boundary Structure and Kinetics, American Society for Metals (1980) p. 297.

[3] Pope D. P. and Wilkinson D. S., Creep and Fracture of Engineering Materials and Structures, eds. Wilshire B. and Owen D. R. J., Pineridge Press, Swansea, U.K. (1.981) p. 531 . 
[4] Recrystalization of Metallic Materials, ed. F. Haessner, Dr. Riederer Verlag Gmb̈H, Stuttgart (1978).

[5] McLean D., J. Physique 36 (1975) C4-273.

[6] McMahon C. J., Jr., Mat. Sci. Eng. 25 (1976) 233.

[7] Stein D. F. and Heldt L. A., Interfacial Segregation, eds. Johnson W. C. and Blakely J. B., American Society for Metals (1979) p. 239.

[8] McLean D., Grain Boundaries in Metals, Oxford Univ. Press, London (1957).

[9] Hondros E. D. and Seah M. P., Int. Metals Rev. (1977) 262.

[10] Guttmann M. and McLean D., Interfacial Segregation eds. Johnson W. C. and Blakely J. M., American Society for Metals (1979) p. 261.

[11] Aust K. T., Can. Met. Quart. 13 (1974) 133.

[1 2] Powe11 B. D. and Woodruff D. P., Phil. Mag. 34 (1976) 169.

[13] Ogura T., McMahon C. J., Jr., Feng H. C. and Vitek V., Acta Meta11. 26 (1978) 1317.

[14] Grovenor C. R. M. and Rae C. M. F., Scripta Meta11. 15 (1981) 1305.

[15] Watanabe T., Murakami T. and Karashima S., Scripta Meta11. 12 (1978) 361.

[16] Suzuki S., Abiko K. and Kimura H., Scripta Metall. 15 (1981) 1139.

[17] Rellick J. R., McMahon C. J., Jr., Marcus H. L. and Palmberg P. W., Metall. Trans. A2 (1971) 1492.

[18] Pichard C., Rieu J. and Goux C., Mém. Scient. Revue Meta11. 70 (1973) 13.

[19] Donald A. M. and Brown L. M., Acta Metall 27 (1979) 59.

[20] Santer H., Gleiter H. and Baro G., Acta Meta11. 25 (1977) 467.

[21] Balluffi R. W., Interfacial Segregation, eds. Johnson W. D. and Blakely J. M., American Society for Metals (1979) p. 193.

[22] Briant C. L. and Messmer R. T., Phil. Mag. 42 (1980) 569.

[23] Messmer R. T. and Briant C. L., Acta Meta11. 30 (1982) 457.

[24] Losch W., Acta Meta11. 27 (1979) 1885.

[25] Anda E., Losch W., Majlis N, and Ure J., Acta Meta11. 30 (1982) 611.

[26] Weins M. J. and Weins J. J., J. Physique 36 (1975) C4-81.

[27] Dahl R. E., Beeler J. R. and Bourquin R. D., Interatomic Potentials and Simulation of Lattice Defects, eds. Gehlen P. C., Beeler I. R. and Jaffee R. I., Plenum Press, New York (1972) p. 673.

[28] Beeler J. R., Dahl R. E. and Bourquin R. D., J. Physique 36 (1975) C4-97.

[29] Nichols S., Scripta Meta11. 15 (1981) 423.

[30] Machlin E. S. and Levi A., Scripta Metal1. 14 (1980) 127.

[31] Machlin E. S., Acta Meta11. 22 (1974) 95, 109, 367, 1433. 
[32] Chang H. K., Lee J. K. and Stein D. F., Interatomic Potentials and Crystalline Defects, ed. Lee J. K., AIME (1981) p. 373.

[33] Hashimoto M., Ishida Y., Yamamoto R., Doyama M. and Fujiwara T., Scripta Metal1. 16 (1982) 267.

[34] Maeda K., Vitek V and Sutton A. P., Acta Meta11. 30 (1982) to be published.

[35] Sutton A. P. and Vitek V., Acta Meta11, 30 (1982) to be published.

[36] Ashby M. F., Spaepen F. and Williams S., Acta Meta11. 26 (1978) 133.

[37] Pond R. C., Smith D. A. and Vitek V., Scripta Metal1. 26 (1978) 699.

[38] Vitek V., Sutton A. P., Smith D. A. and Pond R. C., Grain Boundary Structure and Kinetics, American Society for Metals (1980) p. 115.

[39] Bernal J. D., Proc. Roy. Soc. A280 (1964) 299.

[40] Weins M. J., Chalmers B., Gleiter H. and Ashby M. F., Scripta Meta11. 3 (1969) 601.

[41] Pond R. C. and Vitek V., Proc. Roy. Soc. A357 (1977) 453.

[42] Sutton A. P. and Vitek V., Scripta Metal1. 14 (1980) 129.

[43] Sutton A. P., Ph.D Thesis, Univ, of Pennsylvania (1981).

[44] Sutton A. P. and Vitek V., Phil. Trans. R. Soc. (1982) to be published.

[45] Sutton A. P., Phi1. Mag. (1982) to be published.

[46] Seah M. P. and Hondros E. D., Proc. Roy. Soc. 335A (1973) 191.

[47] Pond R. C. and Smith D. A., Can. Met. Quart. 13 (1974) 39. 


\section{DISCUSSION}

C.L. BRIANT : I assume based on your introduction that you do not fee1 chemistry plays a role in determining the structure and energy of a segregated grain boundary? Could you coment in this point?

V. VITEK : Chemistry certainly plays a role and if we wish to compare behaviour of specific alloys and others, for example, the correct numerical values of the segregation energy, chemistry, i.e. electron theory of bonding, has to be employed. However, the purpose of our study is to investigate various possibilities of the effects of structure upon segregation and this, I believe, can be modelled using a simpler and rather flexible approach which employs pair interactions.

J.D. BUDAI : Have you extended the concept of favoured and non-favoured boundaries to include a variation of grain boundary parameters other than just the misorientation angle?

V. VITEK : Variation of the orientation of the boundary plane has been taken into account by combining the structural unit model with Wulf plot studies (Sutton and Vitek, Phil. Trans. Roy. Soc., to be published). 